\title{
Pre-Chelates, Post-Adducts and Implexes: A perspective proposal for the future of medicinal chemistry
}

\author{
Mario Gosalvez \\ Laboratory and Service of Experimental Biochemistry, Clínica and Hospital Universitario Puerta de Hierro, Madrid and \\ Majadahonda, Comunidad de Madrid, Spain \\ Email: dmg.secre@gmail.com
}

Received 18 September 2013; revised 25 October 2013; accepted 12 November 2013

Copyright (C) 2013 Mario Gosalvez. This is an open access article distributed under the Creative Commons Attribution License, which permits unrestricted use, distribution, and reproduction in any medium, provided the original work is properly cited.

\begin{abstract}
The title of this little assay coins three new words: Pre-Chelates, Post-Adducts and Implexes. These, very daring new excursions into the scientific English language, come from the developments of Quelamycin and Sodium L-Thioproline, through our different group studies arriving to cancer reversal by a dual strategy. It is enough to read all our published materials, cites and related papers, dealing more or less with the chemistry and possible mechanism of action of these compounds, to gather somewhat what signified our monomeric-triferric-doxorubicine, as possibly one of the first Pre-Chelates and our Sodium L-Thioproline, probably as one of the first Post-Adducts. Both molecules come to the human patients with cancer blood from the exterior. After this indication, it will be proposed a seed concept of a new type of molecules, the "Implexes" in which the metals will be embraced instead of being linked.
\end{abstract}

Keywords: New Chelates and Adducts; Embracing Metals, Future of Medicinal Therapeutics; Quelamycin; Thioproli

\section{INTRODUCTION}

In a series of recently published mini-reviews in open journals, we have cited the more important references in our contributions to Quelamycin and Thioproline and its possible utility for cancer reversal by dual strategy and cancer as a therapeutic agent [1-4]. It is enough to read these texts, their therein cites and its related papers to be well aware of what these new molecules would represent as one of the first Pre-Chelates and Post-Adduct, respectively.

Let me please finish, for the moment, these two possible chapters of the future medicinal chemistry by saying

"Former Head. (1970-2010) that the different Pre-Chelates and Post-Adducts, of important molecules of the past, among perhaps our contributions on Quelamycin and Thioproline are pioneering efforts [1-4]. At present, and in the future, it could be more or less evolved aiming to further real advances of the curative medicine and surgery of the times to come.

Thus, having left already these two balls in the fields of biological chemistry experts, I will dedicate this very short article mainly to the Proposal Perspective of "Implexes". A word that, I coined decades ago as a concept not yet considered by biological chemistry. It would be very crucial that the more attentive scrutiny of my following stuttering discourse will come from those more projective experts in advanced chemistry. Their ages are not important.

I am truly just a medical doctor with some scientific experience, who is always trying to help the suffering patient of any disease looking for definitive cures.

\section{IMPLEXES}

I certainly think that, to really attempt to convey to chemical biology experts, with enough clarity and simplicity, what could represent with decades ahead of work a new step forward in curative physiological pharmacology, I would need, if you permit me please, some philosophy of chemistry. That is to say to search for the deeper concepts that lie in the humanity wanting to know of what all is made at the end. These sciences have arrived nowadays, on one hand, at the subatomic chemistry that so far has resulted in atomic particles physics and mathematical diverse plays with the intangible. On the other hand, the molecular neuropharmacology has arrived to psychotropic chemical molecules with several effects on human emotional and mental traits.

\section{A VERY WIDE DEFINITION OF CHEMISTRY}

Without needing to resort to its origins in Alchemy, 
chemistry searched first to define the inner composition of samples of inert matter most calling the attention of human society. Latter arrived to define the chemical structure of organic compounds of importance for life. Urea, Penicillin and Insulin name three of the early great efforts. These are some among the first milestones of chemical synthesis together with Chlorophyll.

With the progressive development of biochemistry, in the last century, the chemistry of life has started to show the immense variety of molecular compositions with higher sense, utility and possibly finality. In some case the simplicity of the solution by nature of the utmost complex task had some biochemists saying to the chemists:

Do you think that such enormous beauty of molecule would have been arrived at, just by chance, necessity and of natural blind evolution?

In the next decades of the 21st siècle chemical sciences could reach however the right molecules to control definitively diseases without secondary toxicities by chemicals so far of impossible synthesis in the laboratory. Of course, first in humans and after in those animals and vegetables more related to our society. I think that the Earth could arrive in few centuries to collaborate with the unicellular beings of phytoplankton and zooplankton. These are the massive and important components of life in our planet for the permanence of life and humanity at large.

\section{4. "IMPOSSIBLE" CHEMICAL SYNTHESIS?}

I think that the better conditions for chemical synthesis invented by Nature are shown by the living cells and organisms. There are many thousands different type of cells for different tasks, either as single organisms, cellular aggregates or forming the tissues of a more complex living beings. For impossible chemical synthesis for our present technology I propose to copy as properly as possible the living cells conditions for synthesis of chemical biological molecules.

What are essentially these conditions?

They are very varied. Life can perform chemical synthesis of complex molecules from very low to very high temperatures. In the complete darkness or with the concourse of light. In very varied atmospheric gas conditions, nutrient's availability, as it is adequate for survival and adaptability including incredible levels of salinity or methane concentration.

My group studied, decades ago, an assortment of complex chemical compounds in its inhibitory effect in rodent mitochondria, extracted and purified from plants of the Canary Islands [5,6]. Many of these chemical structures caused a complete reverence for Mother Nature capacities for chemical synthesis in plants, even to experts in natural compounds.

One of the important things to mention is that the living cell interior's constitute a "soup very heavy". Even water concentration, that is the more abundant simple component of the living cell, rounds 55 Molar in the liver rodent cells. Therefore as the pressure of ambient compounds in this interior is very variegated the competing enzymes must be very specific. The succession of precursor molecules of the compound aimed must be specifically tailored for the final task. The due thinking of this concept needs a careful joining of the more complete experience in biological and organic chemical synthesis.

\section{POSSIBLE SUGGESTIONS FOR ADVANCED CHEMICAL SYNTHESIS?}

I would suggest to try chemical synthesis in very thick mesophases, changing amply the pressure, magnetic field, temperature variations, ambient compounds, gases and enzymes as need for the tasks programmed. The enzymes first should be of vegetables or from unicellular beings, those already known and perhaps the majority to be searched in the next decades. Many other things could I tell if counting with an appropriate collaboration in a international economical effort to copy, doing things first very artificially, life's incredible simplest molecules with great benefit for the future curative pharmacology of all of us together better health

\section{A CONCISE DEFINITION OF “IMPLEXES"}

Instead of linking a metal with adequate chemical chelating forceps, please try to embrace it, and leave it freely in its "Molecular Nest". With it being capable of course to react by, let us say, at this early step of my stuttering thinking: Chemical Resonance.

To say, any word more in this step will compromise the presentation of a generic patent by the main Industries possibly interested in this new research adventure. This is not a journal to use cryptic chemical quantum physics notions to disguise things to experts somewhat lesser advanced in chemistry. Let however end with a tender blink of my left eye:

"Chemical pertaining does not necessarily require chemical bonds and can lead to a set forming part of one set, or two sets, or more sets. It seems a contradiction but perhaps these are situations very common in Life's Astonishing Perfections" in my regard.

\section{REFERENCES}

[1] Gosalvez, M. (2013) Reversal of cancer by dual strategy? Journal of Cancer Therapy, 4, 518-520. 
http://dx.doi.org/10.4236/jct.2013.42064

[2] Gosalvez, M. (2013) Metabolic control of respiration and glycolysis of tumoral cells. Advances in Biological Chemistry, 3, 86-89.

http://dx.doi.org/10.4236/abc.2013.31011

[3] Gosalvez, M. (2013) Cancer as a therapeutic agent? Journal of Cancer Therapy. In press.

[4] Gosalvez, M. (2013) Methods to be developed for some first applications of mitochondrial filamentation. Open
Journal of Biophysics, 3, 51-53.

http://dx.doi.org/10.4236/ojbiphy.2013.31A006

[5] Gosalvez, M., et al. (1976) A screening for selective anticancer agents among plant respiratory inhibitors. European Journal of Cancer, 12, 1003-1009. http://dx.doi.org/10.1016/0014-2964(76)90067-0

[6] Gosalvez, M., et al. (1976) Effects and specificity of anticancer agents on the respiration and energy metabolism of tumor cells. Cancer Treatment Reports, 60, 1-7. 